\title{
Theoretical and experimental models for the synthesis of single-walled carbon nanotubes and their electrochemical properties
}

\begin{abstract}
A major challenge in the field of selective synthesis of single-walled carbon nanotubes (SWCNTs) via chemical vapor deposition (CVD) method is lack of established theoretical model for direct selection of metal/support catalyst to grow the corresponding SWCNTs. This has limited the application of these materials, especially, in electronics. In this report, we introduced circumferential and axial distortions in the Extended Tight Binding (ETB) equations to generate our model equations which correlated the numerical magnitude of chiral index $(n, m)$ of SWCNTs directly with mass fractions of metal/support catalyst matrix, respectively. Theoretical predictions of our model equations showed acceptable deviations with ETB model, and two Fe2O3/A12O3 catalysts were prepared according to this model to grow corresponding SWCNTs $(10,7)$ and $(8,8)$ via CVD pyrolysis of C6H14/N2 feedstock. High-resolution transmission electron microscopy analysis revealed bundled SWCNTs while analysis of their Raman profiles showed consistency with the radial breathing modes, diameter and energy band gaps of SWCNTs $(10,7)$ and $(8,8)$. Electrochemical analysis of the samples suggested potentials as pseudocapacitor electrodes. If fully explored and optimized, this model may complement or augment the existing in-situ epitaxial growth model.
\end{abstract}

